\title{
Surgical results of proximal gastrectomy for early-stage gastric cancer: jejunal interposition and gastric tube reconstruction
}

\author{
Yosuke Adachi, Tokuji Inoue, Yoshiaki Hagino, Norio Shiraishi, Katsuhiro Shimoda, and Seigo Kitano \\ First Department of Surgery, Oita Medical University, 1-1 Idaigaoka, Hasama-machi, Oita 879-5593, Japan
}

\begin{abstract}
:
Background. The frequency of tumors in the upper one-third of the stomach has been increasing. The standard operation for proximal gastric cancer has been total or proximal gastrectomy. The aim of this study was to present the pathologic and surgical results of 30 patients with early-stage proximal gastric cancer managed by proximal gastrectomy. Methods. A consecutive series of 30 patients who underwent proximal gastrectomy for early-stage proximal gastric cancer was studied. Sixteen patients underwent jejunal interposition, while 14 underwent gastric tube reconstruction, which consisted of a direct anastomosis between the esophagus and the remnant of the tube-like stomach.

Results. Twenty patients $(67 \%)$ had no abdominal symptoms and the lesions were detected by screening gastric fiberscopy. The tumors were mostly located along the lesser curvature (73\%), were grossly depressed type (IIc) $(\mathbf{7 0} \%)$, and histologically well differentiated type $(63 \%)$. The depth of wall invasion was the mucosa in 12 patients, submucosa in $\mathbf{1 5}$, and muscularis propria in 3; lymph node metastasis was absent in 28 patients $(93 \%)$. When compared with patients with jejunal interposition, patients with gastric tube reconstruction had a shorter operation time (327 vs $165 \mathrm{~min})$, less blood loss (508 vs 151 g), and shorter hospital stay after operation (31 vs 17 days). Endoscopy and 24-h pH monitoring showed no evidence of reflux esophagitis, except in 1 patient with gastric tube reconstruction, and no patient died of recurrence.

Conclusions. Early-stage proximal gastric cancer can be successfully treated by proximal gastrectomy. Since gastric tube reconstruction is a simple, easy, and safe procedure, proximal gastrectomy followed by gastric tube reconstruction is recommended for patients with early-stage proximal gastric cancer.
\end{abstract}

Offprint requests to: $\mathrm{Y}$. Adachi

Received for publication on Jan. 5, 1999; accepted on Feb. 10, 1999
Key words: stomach, cancer, gastric cancer, proximal gastric cancer, proximal gastrectomy, jejunal interposition, gastric tube

\section{Introduction}

The frequency of tumors in the upper one-third of the stomach has been increasing [1]. Compared with patients having distal gastric cancer, those having proximal gastric cancer have a high operative mortality and low survival rate [2]. The standard operation for cancer of the upper one-third of the stomach has been total gastrectomy followed by Roux-en-Y reconstruction using a jejunal loop. Recently, advances in diagnostics and techniques have increased the number of detections of early-stage proximal gastric cancer [3]. In such patients, some Japanese authors stress the usefulness of proximal gastrectomy reconstructed by jejunal interposition or jejunal pouch reconstruction $[4,5]$.

Clinicopathologic studies indicate that cancers located in the upper one-third of the stomach are characterized by an advanced stage of the disease and by poor prognosis, even after radical gastrectomy and lymphadenectomy $[2,6]$. In contrarst, early cancers in the upper stomach show the same biologic behavior as those in the lower stomach, and surgical results of patients with early-stage upper gastric cancer are excellent [7]. However, to our knowledge, there is no comprehensive study concerning the pathology and outcome of early-stage gastric cancer treated by proximal gastrectomy.

We present the clinicopathologic data and surgical results of 30 patients with early-stage proximal gastric cancer managed by proximal gastrectomy, and introduce a simple and safe technique of reconstruction using a gastric tube [8]. 


\section{Patients and methods}

We studied a consecutive series of 30 patients who underwent proximal gastrectomy for early-stage cancer located in the upper one-third of the stomach at the First Department of Surgery, Oita Medical University, from 1992 to 1998. Sixteen patients treated before February 1997 underwent jejunal interposition, and 14 patients treated after February 1997 had gastric tube reconstruction.

Information on the age and sex of patients, symptoms, findings of gastric fiberscopy, method of detection of the lesion, operative complications, body weight, and hospital stay after operation was obtained from medical charts. Data on operation time and blood loss were obtained from operation records. The location, size, gross type, and histologic type of tumors, depth of wall invasion, and presence or absence of lymph node metastasis were obtained from histopathology reports. These clinicopathologic findings were based on the Japanese classification of gastric carcinoma outlined by the Japanese Gastric Cancer Association [9].

Proximal gastrectomy with gastric tube reconstruction consisted of the following procedures [8]: (1) a laparotomy was made through an upper midline skin incision, (2) the upper two-thirds of the stomach and abdominal esophagus was fully mobilized, (3) the esophagus was transected and a pursestring suture was placed, using 3-0 Proline, (4) the anvil of a circular stapler (Proximate ILS 25; Ethicon Endo-Surgery, Inc., Cincinnati, $\mathrm{OH}, \mathrm{USA}$ ) was introduced into the esophagus and the pursestring suture was tied, (5) the stomach was transected between the points of the distal three-fourths of the lesser curvature and half of the greater curvature, using an autosuture stapler (GIA 60; United States Surgical, Inc., Norwalk, CT, USA) three times (Fig. 1a), (6) the circular stapler was inserted through a small opening of the stapled line and its center rod was pierced through the anterior wall near the top of the gastric tube (Fig. 1b), (7) after the center rod was combined with the anvil shaft, the circular stapler was fired, (8) the opening of the gastric tube was closed in two layers and a pyloroplasty was carried out by the Heineke-Mikulicz or Aust method, (9) the abdomen was closed in the usual manner. Thus, the upper two-thirds of the stomach and regional lymph nodes (No. 1, 2, 3, 4sa, 4sb, 7) were removed, and a gastric tube measuring about $15 \mathrm{~cm}$ in length and $3 \mathrm{~cm}$ in width was made (Fig. 1c,d).

Ambulatory 24-h pH monitoring was carried out using an esophageal $\mathrm{pH}$ probe introduced through the nose into the esophagus and positioned $3 \mathrm{~cm}$ above the esophagogastric anastomosis site. Esophageal $\mathrm{pH}$ changes were recorded by a Digitrapper MK III (Synectics Medical, Stockholm, Sweden) and an EsopHogram (Gastrosoft, Dallas, TX, USA). During the examination, the patients carried on their normal life activities and were allowed their usual diets.

Operation time, blood loss, weight loss, and hospital stay after operation were compared statistically in the patients with jejunal interposition and those with gastric tube reconstruction, using the $t$-test.

\section{Results}

The age of the patients ranged from 37 to 88 years, (mean, 68 years); 25 (83\%) were men and 5 were women (Table 1). Twenty patients $(67 \%)$ had no abdominal symptoms and their lesions were detected by screening gastric fiberscopy. Ten patients had had epigastralgia, and 6 of these had grossly ulcerated cancer (III type). On endoscopy, 21 lesions were depressed or ulcerated and 7 were elevated. Two flat lesions were detected because of mucosal redness. The lesions were mostly located along the lesser curvature (73\%), and the mean distance between the esophagogastric junction and the oral edge of the tumor was $2.1 \mathrm{~cm}$. The size of tumors ranged from 1.3 to $5.0 \mathrm{~cm}$ (mean, $2.5 \mathrm{~cm}$ ). Predominant gross and histologic types were IIc (70\%) and well differentiated tubular $(53 \%)$ or papillary $(10 \%)$ adenocarcinoma, respectively. Depth of wall invasion was the mucosa in 12 patients, submucosa in 15 , and muscularis propria in 3; lymph node metastasis was absent in 28 patients ( $93 \%$ ).

When compared with patients with jejunal interposition, patients with gastric tube reconstruction had shorter operation time, less blood loss, and shorter hospital stay after operation (Table 2). There was no anastomotic leakage or intraabdominal abscess, and no operative deaths. One patient with laparoscopic proximal gastrectomy and one patient with conventional proximal gastrectomy showed anastomotic stenosis and needed dilatation by endoscopic bougie. One patient who underwent laparoscopic proximal gastrectomy with gastric tube reconstruction complained of heartburn and had a mild degree of esophagitis on endoscopy, but was successfully treated with antacids. The other 27 patients recovered uneventfully and did not complain of heartburn or retrosternal pain suggesting gastroesophageal reflux disease. Endoscopy was easily done in patients with gastric tube reconstruction, and showed no evidence of esophagitis, gastritis, or stomal ulcer.

Nine patients with gastric tube reconstruction were tested by ambulatory 24-h $\mathrm{pH}$ monitoring of the esophagus. Although the above-mentioned patient with mild esophagitis had a $\mathrm{pH}<4.0$ holding time of $261 \mathrm{~min}$ $(18 \%)$, in the other eight patients, the $\mathrm{pH}$ of the lower esophagus was between 5 and 8 during the day and night, and there was no evidence of acid or alkaline reflux into the esophagus. 


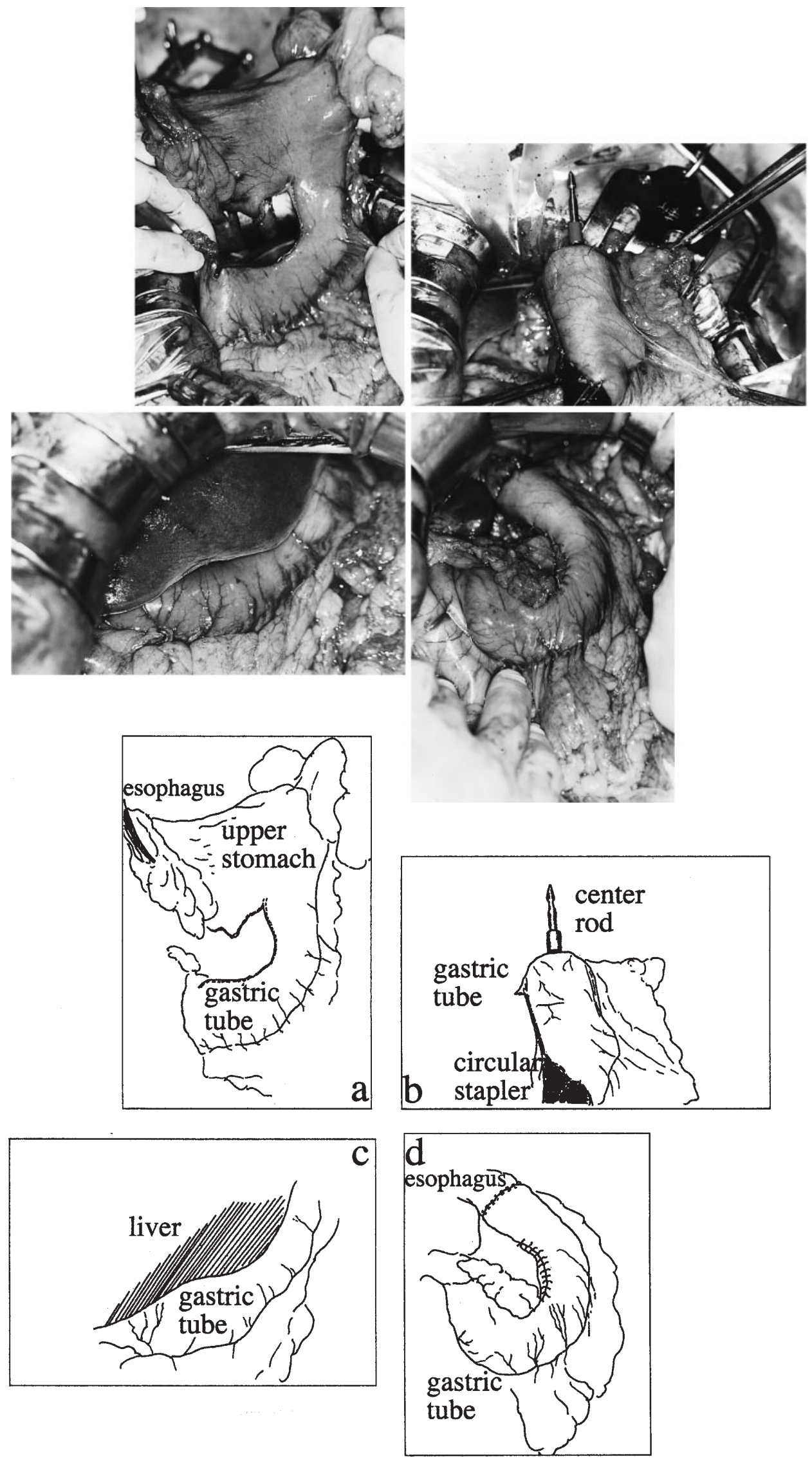

Fig. 1. a Stomach is transected between the points of the distal three-fourths of the lesser curvature and half of the greater curvature, using an autosuture stapler (GIA 60; US Surgical) three times. b A circular stapler is inserted through a small opening of the stapled line and its center rod is pierced through the anterior wall near the top of the gastric tube. c, d Proximal gastrectomy is reconstructed with a gastric tube measuring about $15 \mathrm{~cm}$ in length and $3 \mathrm{~cm}$ in width 
Table 1. Early-stage gastric cancers treated with proximal gastrectomy

\begin{tabular}{|c|c|}
\hline & Proximal gastrectomy $(n=30)$ \\
\hline \multicolumn{2}{|l|}{ Age, years } \\
\hline Range / Mean & $37-88$ / 68 \\
\hline \multicolumn{2}{|l|}{ Sex } \\
\hline Male / Female & $25(83) / 5(17)$ \\
\hline \multicolumn{2}{|l|}{ Symptoms } \\
\hline Absent / Epigastralgia & $20(67) / 10(33)$ \\
\hline \multicolumn{2}{|l|}{ Gastroscopic findings } \\
\hline Depressed / Ulcerated / Flat / Elevated & $12(40) / 9(30) / 2(7) / 7(23)$ \\
\hline \multicolumn{2}{|l|}{ Location of tumor } \\
\hline Lesser / Posterior / Anterior & $22(73) / 5(17) / 3(10)$ \\
\hline \multicolumn{2}{|l|}{ Distance from EGJ, cm } \\
\hline Range / Mean & $0.0-6.0 / 2.1$ \\
\hline \multicolumn{2}{|l|}{ Size of tumor, $\mathrm{cm}$} \\
\hline Range / Mean & $1.3-5.0 / 2.5$ \\
\hline \multicolumn{2}{|l|}{ Gross type } \\
\hline IIc $(+$ III $) /$ IIb / IIa $(+$ IIc $) /$ I & $21(70) / 1(3) / 5(17) / 3(10)$ \\
\hline \multicolumn{2}{|l|}{ Histologic type } \\
\hline Pap / Tub / Poor / Sig / Muc & $3(10) / 16(53) / 6(20) / 4(13) / 1(3)$ \\
\hline \multicolumn{2}{|l|}{ Depth of wall invasion } \\
\hline Mucosa / Submucosa / Muscularis & $12(40) / 15(50) / 3(10)$ \\
\hline \multicolumn{2}{|l|}{ Lymph node metastasis } \\
\hline Absent / Present & $28(93) / 2(7)$ \\
\hline
\end{tabular}

Numbers in parentheses are percentages. EGJ, Esophagogastric junction; pap, papillary; tub, tubular; poor, poorly differentiated; sig, signet-ring cell; muc, mucinous

Table 2. Jejunal interposition and gastric tube reconstruction

\begin{tabular}{|c|c|c|c|}
\hline & Jejunal interposition $(n=16)$ & Gastric tube $(n=14)$ & $P$ value \\
\hline \multicolumn{4}{|l|}{ Operation time, $\min$} \\
\hline Mean $\pm \mathrm{SD}$ (range) & $326.9 \pm 70.6(215-463)$ & $164.9 \pm 38.3^{\mathrm{a}}(120-211)$ & $<0.01$ \\
\hline \multicolumn{4}{|l|}{ Blood loss, $g$} \\
\hline Mean $\pm \mathrm{SD}$ (range) & $508.4 \pm 318.1(155-1215)$ & $151.1 \pm 99.6^{\mathrm{a}}(50-360)$ & $<0.01$ \\
\hline \multicolumn{4}{|l|}{ Hospital stay, days } \\
\hline Mean \pm SD (range) & $30.5 \pm 8.6(18-52)$ & $16.7 \pm 3.4^{\mathrm{a}}(14-22)$ & $<0.01$ \\
\hline \multicolumn{4}{|l|}{ Weight loss, $\mathrm{kg}$} \\
\hline Mean \pm SD (range) & $5.99 \pm 2.91(2.0-12.0)$ & $4.21 \pm 1.03(2.4-5.5)$ & 0.0537 \\
\hline \multicolumn{4}{|l|}{ Complications } \\
\hline Anastomotic leakage & 0 & 0 & \\
\hline Intraabdominal abscess & 0 & 0 & \\
\hline Pancreatic fistula & 0 & 0 & \\
\hline Anastomotic stenosis & 1 & 1 & \\
\hline Reflux esophagitis & 0 & 1 & \\
\hline Operative death & 0 & 0 & \\
\hline
\end{tabular}

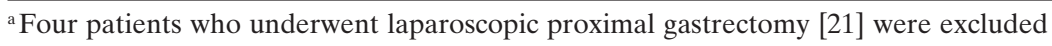

Twenty-nine patients were alive without recurrence during a follow-up period of 1-76 months (mean, 27 months); one patient who had had liver cirrhosis died of liver failure 12 months after operation.

\section{Discussion}

Total gastrectomy has been indicated for patients with advanced cancers located in the upper third of the stom- ach, and proximal gastrectomy has been employed for those with early-stage proximal gastric cancers. Of the 30 patients in our series, 25 had tumors restricted to the mucosa or to the submucosa without lymph node metastasis, and 5 had either invasion down to the muscularis propria or metastasis to the regional lymph nodes. This clinicopathologic study clarified that earlystage gastric cancers treated by proximal gastrectomy were characterized by: occurrence in the aged, predominance in men, location along the lesser curvature, 
grossly depressed or ulcerated lesions, and histologically well differentiated type. Lymph node metastasis was rare and the prognosis of the patients was excellent.

Several authors have studied the clinicopathologic features of proximal gastric cancer. Calan et al. [10] evaluated the surgical results of 91 patients and showed that the tumor was resected in $57(63 \%)$, gastrectomy was curative in $28(31 \%)$, and their 5-year survival rate was low (40\%). Rohde et al. [2] indicated that advanced cancers (TNM stages IIIb and IV) were more frequent in proximal than in distal gastric cancers (60\% vs $38 \%$ ) and poorer long-term outcome was evident in proximal gastric cancer for all TNM stages. Maehara et al. [6] demonstrated that proximal gastric cancers were characterized by large size, infiltrative growth, frequent serosal invasion, lymph node metastasis, liver metastasis, and lower survival rate. These authors concluded that the only way to improve survival was to detect the cancer at an early stage and to increase the curative resection rate.

Recently, Harrison et al. [11] clarified that although the overall 5-year survival rate was different between patients with proximal and distal gastric cancers $(42 \%$ vs $61 \%$ ), the survival rate was not significantly different between the two groups for each stage. Patients with gastroesophageal junction tumors had the lowest 5-year survival rate $(22 \%)$, showing that surgical results had a worse outcome as the tumor was located more proximally. Although Stipa et al. [12] showed that adenocarcinoma of the gastric cardia was far advanced when diagnosed in most patients, and the 5-year survival rate after resection was $17 \%$, Mori et al. [3] reported that only 1 of 21 patients with early cancer of the gastric cardia died of recurrence during a mean follow-up period of 64 months. Thus, when diagnosed at an early stage, cancer of the gastric cardia was successfully controlled by gastrectomy.

The type of operation to be performed for proximal gastric cancer is still controversial. Kaibara et al. [13] indicated that none of 90 tumors confined to the upper third of the stomach had metastasis to the pyloric lymph nodes, and the 5-year survival rate after curative resection was not different between proximal and total gastrectomies (57\% vs 58\%); they concluded that proximal gastrectomy was indicated when the tumor was confined to the upper third of the stomach. Kitamura et al. [7] showed that although three tumors invading the serosa had metastatic lymph nodes along the lower part of the stomach, the survival rate was not different between proximal and total gastrectomies; they advocated that proximal gastrectomy should be performed when the tumor invasion was not beyond the muscularis propria.

Harrison et al. [14] demonstrated that the time to recurrence and 5-year survival rate were not different between proximal and total gastrectomies (16 months vs 18 months, $43 \%$ vs $41 \%$ ), and concluded that total gastrectomy was not necessary for proximal gastric cancer. For early cancer, Furukawa et al. [15] reported that fundectomy, when compared with total gastrectomy, showed less blood loss ( $300 \mathrm{ml}$ vs $555 \mathrm{ml})$ and less frequent pancreatic fistula ( $0 \%$ vs $15 \%$ ), and they stated that limited resection was feasible in patients with early proximal gastric cancers. Thus, proximal gastrectomy has become the treatment of choice for patients with proximal gastric cancer, especially when the tumor invasion is not beyond the muscularis propria.

There are several methods for reconstruction after proximal gastrectomy. Since direct anastomosis between the esophagus and the remnant stomach resulted in severe reflux esophagitis, jejunal interposition or Roux-en-Y esophagojejunostomy with side-to-end jejunogastrostomy (double-tract method) has been employed. Recently, gastric substitutes with sufficient capacity for food have been made by the interposition of a jejunal pouch $[4,5,16]$ or a short segment of left colon [17]. Although these gastric substitutes are reported to be useful, the procedures are complicated and timeconsuming, and need several stapling devices. Furthermore, a length of about $40 \mathrm{~cm}$ of upper jejunum, which is responsible for the digestion and absorption of nutrients and minerals, must be sacrificed to make a jejunal pouch, and the long-term nutritional effects of these procedures are not known.

Our technique is an application of intrathoracic reconstruction in which a gastric tube was used after thoracic esophagectomy for esophageal cancer [18]. De spite negative pressure in the thoracic cavity, gastric tube reconstruction after esophagocardiectomy for cancer of the gastric cardia or abdominal esophagus does not result in reflux esophagitis. In this study, although one patient had mild reflux esophagitis, the remaining 13 patients with gastric tube reconstruction showed no evidence of reflux esophagitis with symptoms, or on endoscopy and 24-h pH monitoring [19].

In our series, two patients had lymph node metastasis. One patient, a 76-year-old man, had a IIc-type tumor measuring $4.1 \mathrm{~cm}$ in diameter. Poorly differentiated adenocarcinoma had invaded the submucosa and metastasized to one lymph node at the right paracardia (No. 1). The remaining 14 lymph nodes, including those around the left gastric artery (No. 7), were negative. Therefore, it was considered that the tumor tissue had been completely removed by proximal gastrectomy with gastric tube reconstruction. The other patient, an 83-year-old woman, had a IIc-type tumor measuring $3.7 \mathrm{~cm}$ in diameter. Signet-ring cells had invaded the submucosa and involved 9 of 16 lymph nodes dissected (No. 1, 1 of 1 ; No. 3, 8 of 10). The cancer cells were found in the sinusoids within the lymph nodes. This 
unusual patient was treated by proximal gastrectomy with gastric tube reconstruction and had no evidence of recurrence at a follow-up period of 6 months. Whether metastasis was present or absent in the lymph nodes along the right gastroepiploic vessels (No. 4d) and suprapyloric and infrapyloric lymph nodes (No. 5, No. 6) was not known, and whether total gastrectomy should have been done was unclear.

In our procedure of proximal gastrectomy with gastric tube reconstruction, three-fourths of the lesser curvature was resected and most lymph nodes along the lesser curvature were removed en bloc, while lymph nodes along the distal half of the greater curvature were left behind. Since most tumors $(73 \%)$ were located along the lesser curvature and no tumors had metastasis to the lymph nodes along the greater curvature, we consider that resection of the proximal half of the greater curvature (No. 4sa, No. 4sb) would be sufficient for most patients with early-stage proximal gastric cancer.

Thus, proximal gastrectomy with gastric tube reconstruction has several advantages: (1) there is only one anastomosis made by a circular stapler and the procedure is technically simple, easy, and safe; (2) the passage of food is good, and reflux esophagitis rarely occurs; and (3) the proximal segment of the jejunum, which is important for nutrient absorption, is well preserved. We believe that proximal gastrectomy followed by gastric tube reconstruction is the procedure of choice for patients with early cancer or Dukes' A cancer [20] located in the upper one-third of the stomach.

Acknowledgments. We thank Mr. H. Yoshida for his excellent photography and Miss Y. Kawano for her technical aasistance.

\section{References}

1. Salvon-Harman JC, Cady B, Nikulasson S, Khettry U, Stone MD, Lavin P. Shifting proportions of gastric adenocarcinomas. Arch Surg 1994;129:381-9.

2. Rohde H, Bauer P, Stützer H, Heitmann K, Gebbensleben B. German Gastric Cancer TNM Study Group. Proximal compared with distal adenocarcinoma of the stomach: differences and consequences. Br J Surg 1991;78:1242-8.

3. Mori M, Kitagawa S, Iida M, Sakurai T, Enjoji M, Sugimachi K, Oiwa T. Early carcinoma of the gastric cardia: a clinicopathologic study of 21 cases. Cancer 1987;59:1758-66.
4. Kameyama J, Ishida H, Yasaku Y, Suzuki A, Kuzu H, Tsukamoto M. Proximal gastrectomy reconstructed by interposition of a jejunal pouch: surgical technique. Eur J Surg 1993;159:491-3.

5. Takeshita K, Saito N, Saeki I, Honda T, Tani M, Kando F, Endo M. Proximal gastrectomy and jejunal pouch interposition for the treatment of early cancer in the upper third of the stomach: surgical techniques and evaluation of postoperative function. Surgery 1997;121:278-86.

6. Maehara Y, Moriguchi S, Kakeji Y, Orita H, Haraguchi M, Korenaga D, et al. Prognostic factors in adenocarcinoma in the upper one-third of the stomach. Surg Gynecol Obstet 1991;173: 223-6.

7. Kitamura K, Yamaguchi T, Nishida S, Yamamoto K, Ichikawa D, Okamoto $\mathrm{K}$, et al. The operative indications for proximal gastrectomy in patients with gastric cancer in the upper third of the stomach. Jpn J Surg 1997;27:993-8.

8. Adachi Y, Katsuta T, Aramaki M, Morimoto A, Shiraishi N, Kitano S. Proximal gastrectomy and gastric tube reconstruction for early cancer of the gastric cardia. Dig Surg (in press).

9. Japanese Gastric Cancer Association. Japanese classification of gastric carcinoma: 2nd English ed. Gastric Cancer 1998;1:1024.

10. de Calan L, Portier G, Ozoux JP, Rivallain B, Perrier M, Brizon J. Carcinoma of the cardia and proximal third of the stomach: results of surgical treatment in 91 consecutive patients. Am J Surg 1988;155:481-5.

11. Harrison LE, Karpeh MS, Brennan MF. Proximal gastric cancers resected via a transabdominal-only approach: results and comparisons to distal adenocarcinoma of the stomach. Ann Surg 1997;225:678-85.

12. Stipa S, Giorgio AD, Ferri M. Surgical treatment of adenocarcinoma of the cardia. Surgery 1992;111:386-93.

13. Kaibara N, Nishimura O, Nishidoi H, Kimura O, Koga S. Proximal gastrectomy as the surgical procedure of choice for upper gastric carcinoma. J Surg Oncol 1987;36:110-2.

14. Harrison LE, Karpeh MS, Brennan MF. Total gastrectomy is not necessary for proximal gastric cancer. Surgery 1998;123:12730.

15. Furukawa H, Hiratsuka M, Imaoka S, Ishikawa O, Kabuto T, Sasaki Y, et al. Limited surgery for early gastric cancer in cardia. Ann Surg Oncol 1998;5:338-41.

16. Oka M, Yamamoto K, Nakamura M, Miyahara M, Ueno T, Nishihara K, et al. Reconstruction after proximal gastrectomy using a stapled, U-shaped jejunal pouch. J Am Coll Surg 1998; 186:601-3.

17. Ohwada S, Nakamura S, Sato Y, Iwazaki S, Oriuchi N, Izumi M, et al. Short segment of left colon substitution following proximal gastrectomy. Hepatogastroenterology 1996;43:1256-9.

18. Sugimachi K, Ohno S, Maekawa S, Mori M, Kuwano H, Ueo H. Safety of EEA staplers for anastomosis between upper esophagus and the gastric tube. J Surg Oncol 1990;43:40-4.

19. Shiraishi N, Hirose R, Morimoto A, Kawano K, Adachi Y, Kitano S. Gastric tube reconstruction prevented esophageal reflux after proximal gastrectomy. Gastric Cancer 1998;1:78-9.

20. Adachi Y, Inomata M, Kitano S, Mori M, Maehara Y, Sugimachi K. Dukes' A tumor: new criteria for early gastric cancer. Oncology Reports 1997;4:1235-7.

21. Kitano S, Adachi Y, Shiraishi N, Suematsu T, Bandoh T. Laparoscopy-assisted proximal gastrectomy for early gastric carcinomas: report of two cases. Surgery Today 1999;29:389-91. 